mens to the laboratory. In the test presented here dried specimens were eluted in $4 \mathrm{ml}$ of sample buffer and yet only $200 \mu \mathrm{l}$ is used for $H$ pylori antibody estimation. This gives the opportunity for the unused eluted portion to be used to measure other antibodies as the sample eluted can be kept for at least one week at $4^{\circ} \mathrm{C}$ without loss of activity (results not shown).

Whole blood dried on filter paper provides an ideal method of specimen collection for an expanding range of tests applicable to human health and disease. The stability and reproducibility of the results are comparable with those obtained with whole serum but without the inconvenience of that material.
This filter helper procedure can also be applied to other serological methods.

1 Zhuang H, Coulepis AG, Locarnini SA, Gust ID. Detection of markers of hepatitis $B$ infection in serum dried onto filter paper: an application to field studies. Bull $W H O$ 1982;60:783-7.

2 Guthrie R, Susi A. A simple phenylamine method for detecting phenylketonuria in large populations of new born infants. Pediatrics 1963;32:338-43.

3 Thielmann K, Moreina Aquino A. Whole blood samples dried and stored on filter paper as substrate for the dried and stored on filter paper as substrate for the electrophoretic separation of haemoglobin $S$ from haemoglobin A: A screening procedure. Clin Chim Acta

4 Farzadegan H, Quinn T, Polk F. Detecting antibodies to human immunodeficiency virus in dried blood or filter human immunodeficiency virus in

paper. Infect Dis 1987;155:1073-4.
5 Garcia E, Sotelo J. Storage of cerebrospinal fluid on paper. Lancet 1989; ii: 1046.

6 Kaldor J, McCarthy P, Watson J, Dwyer B. Immune response to Campylobacter pyloridis in patients with peptic ulceration. Lancet 1985;i:269.

\title{
Laboratory detection of ciprofloxacin resistant Neisseria gonorrhoeae
}

\author{
A Turner, A E Jephcott, K R Gough
}

\begin{abstract}
During 1989 and 1990 strains of Neisseria gonorrhoeae with reduced susceptibility to ciprofloxacin were isolated in laboratories across the United Kingdom. Treatment failures were associated with some of these infections. These strains were detected by quantitative susceptibility testing because the zone of inhibition around $5 \mu \mathrm{g}$ ciprofloxacin discs shows little decrease in size even with those that are the most resistant. This study determined that strains with reduced susceptibility to ciprofloxacin (MIC of $\geqslant 0.05 \mathrm{mg} / 1$ ) produced no zone of inhibition around a commercially available disc containing $30 \mu \mathrm{g}$ of nalidixic acid. Ciprofloxacin sensitive (MIC of $<0.05 \mathrm{mg} / 1$ ) strains, however, grew with a large zone (>21 mm) around this disc. These observations suggest that laboratories could adopt this disc test to detect those strains for which ciprofloxacin is not appropriate treatment.
\end{abstract}

Ciprofloxacin has good in vitro activity against most strains of Neisseria gonorrhoeae $e^{1-3}$ and consequently has been increasingly used as a first line treatment for gonorrhoea. Strains with decreased sensitivity (MIC of $\geqslant 0.05 \mathrm{mg} / \mathrm{l}$ ), however, have been detected with increasing frequency in the past year and treatment failures with ciprofloxacin have been associated with some of these infections. ${ }^{45}$

It would clearly be beneficial if these strains could be detected by routine testing in all laboratories. Unfortunately, agar dilution quantitative sensitivities are labour intensive and are not usually practicable in a busy laboratory. At the Gonococcus Reference Unit (GRU), however, a simple qualitative test has been developed which seems to overcome this difficulty and could facilitate screening for these organisms.

\section{Method and results}

All gonococci received by the GRU are sensitivity tested by the agar dilution method to five antibiotics including ciprofloxacin. From these isolates representative ciprofloxacin sensitive strains, with a range of MICs, and all 25 strains with reduced sensitivity available to us (including isolates with ciprofloxacin MICs of up to $0.50 \mathrm{mg} / \mathrm{l}$ ), were selected for this study. Their sensitivity was evaluated in a simple antibiotic disc test on heated blood agar using commercially available ciprofloxacin $(5 \mu \mathrm{g})$ and nalidixic acid $(30 \mu \mathrm{g})$ discs. After overnight incubation the diameters of the zones were measured using calipers.

All less sensitive strains show little decrease in zone of inhibition around the $5 \mu \mathrm{g}$ ciprofloxacin disc, even those with the highest MICs (fig 1). The data in fig 2, however, show that the zone of inhibition around the nalidixic acid disc was reduced to zero for strains with ciprofloxacin MICs greater than $0.025 \mathrm{mg} / \mathrm{l}$, whereas a large $(>21 \mathrm{~mm}$ in diameter) inhibition zone around this disc was invariably present when sensitive strains (MIC of $\leqslant 0.025 \mathrm{mg} / \mathrm{l}$ ) were tested. 


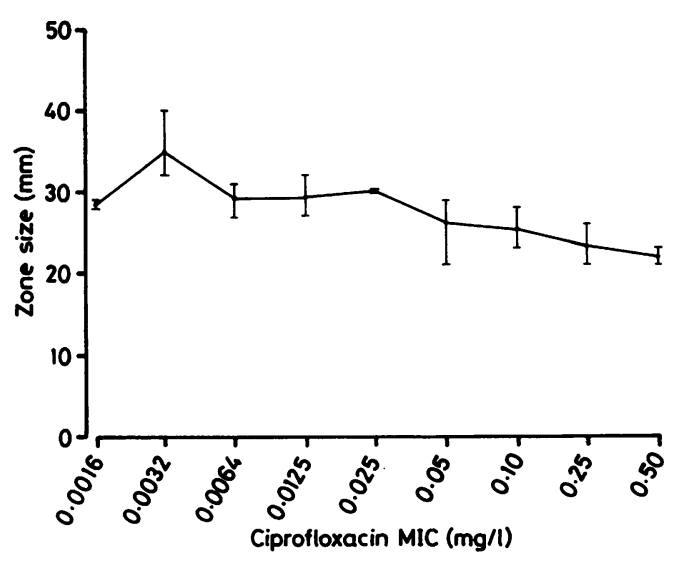

Figure 1 Inhibition zone size around a $5 \mu \mathrm{g}$ ciprofloxacin disc for isolates with the indicated ciprofloxacin MIC. Bars indicate the range of zone size found and the line indicates the mean zone size.

\section{Discussion}

We have shown that the $30 \mu \mathrm{g}$ nalidixic acid disc can be used to provide a good screening test for ciprofloxacin resistance in $N$ gonorrhoeae. The results suggest that laboratories which serve a genitourinary medicine clinic where ciprofloxacin is used could adopt this test to detect those strains for which this drug is not appropriate.

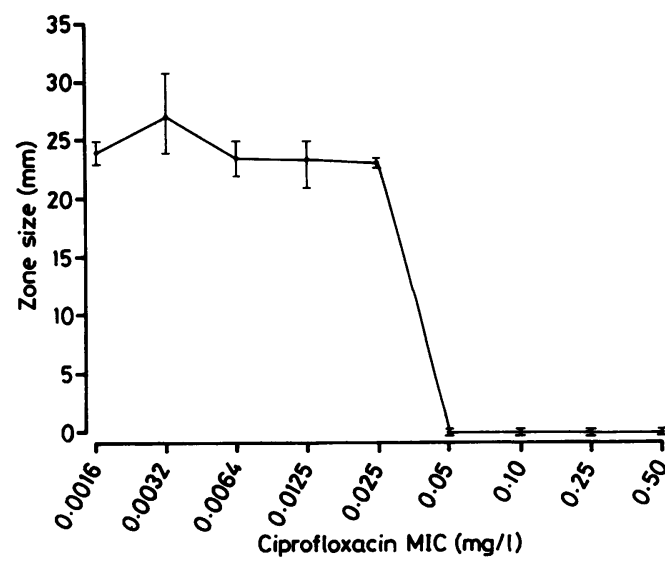

Figure 2 Inhibition zone size around a $30 \mu \mathrm{g}$ nalidixic acid disc for isolates with the indicated ciprofloxacin MIC. Bars indicate the range of zone size found and the line indicates the mean zone size.

1 Lefevre JC, Tempesta MC, Gaubert E, Lareng MB. Invitro activity of six quinolone derivatives against Neisseria gonorrhoeae. Chemotherapy 1988;34:315-7.

2 Klingeren van B, Dessens-Kroon M, Verheuvel M. In-vitro activity of quinolones against penicillinase-producing and non-penicillinase-producing gonococci. Chemioterapia 1985;4(suppl 2):464-5.

3 Melby K, Faegri A. The in-vitro activity of norfloxacin, ofloxacin and ciprofloxacin and other antibiotics in current use against Neisseria gonorrhoeae. APMIS 1989 97:347-50.

4 Gransden WR, Warren CA, Phillips I, Hodges M, Barlow $D$. Decreased susceptibility of Neisseria gonorrhoeae to ciprofloxacin. Lancet 1990;335:51.

5 Jephcott AE, Turner A. Ciprofloxacin resistance in gonococci. Lancet 1990;335:165.

\title{
Examination of spinal cord in diseases of the craniocervical junction and high cervical spine
}

\author{
J F Geddes, A G Gonzalez
}

\author{
Department of \\ Neuropathology, \\ National Hospital for \\ Neurology and \\ Neurosurgery, Maida \\ Vale, London W9 1TL \\ J F Geddes \\ National Hospital for \\ Neurology and \\ Neurosurgery, \\ Queen's Square, \\ London WC1 \\ A G Gonzalez \\ Correspondence to: \\ Dr J F Geddes \\ Accepted for publication \\ 16 August 1990
}

\begin{abstract}
A simple necropsy technique for the removal of the craniocervical junction was devised: a relatively small specimen comprising part of the clivus, the foramen magnum, and cervical vertebral canal is removed in one piece with the medulla and spinal cord inside, and examined systematically after fixation. This method, used in a series of patients with chronic craniocervical instability, allows both good clinicopathological correlations to be made and histological changes in the lower medulla or upper cervical cord segments to be related to sites of extrinsic compression.
\end{abstract}

Chronic medullospinal or high cervical cord compression produces brainstem symptoms and upper cervical myelopathy with long tract signs. Clinicopathological correlations are not often described, because it is relatively difficult to examine the craniocervical junction. Some pathologists remove the entire bony column with the spinal cord inside, but may not include the foramen magnum. This is also a time consuming procedure, creating problems in reconstruction for the mortuary technician. We performed necropsies on 15 patients with atlantoaxial and subaxial dislocation, 13 of whom had had transoral surgery for decompression of the cord, in almost 